Rapport - Société canadienne d'histoire de l'Église catholique

\title{
L'histoire providentielle, morale ou patriotique est-elle légitime?
}

\section{Jean Leclerc}

Volume 30, 1963

URI : https://id.erudit.org/iderudit/1007357ar

DOI : https://doi.org/10.7202/1007357ar

Aller au sommaire du numéro

Éditeur(s)

La Société canadienne d'histoire de l'Église catholique

ISSN

0318-6148 (imprimé)

1927-7075 (numérique)

Découvrir la revue

Citer cet article

Leclerc, J. (1963). L'histoire providentielle, morale ou patriotique est-elle légitime? Rapport - Société canadienne d'histoire de l'Église catholique, 30, 81-87. https://doi.org/10.7202/1007357ar

Tous droits réservés @ La Société canadienne d'histoire de l'Église catholique, 1964
Ce document est protégé par la loi sur le droit d'auteur. L'utilisation des services d'Érudit (y compris la reproduction) est assujettie à sa politique d'utilisation que vous pouvez consulter en ligne.

https://apropos.erudit.org/fr/usagers/politique-dutilisation/ 


\section{L'histoire providentielle, morale ou patriotique est-elle légitime?}

Notre intention, en abordant ce sujet, n'est pas d'entrer dans les controverses qui se sont élevées sur cette question et dans lesquelles chacun a apporté les solutions qui lui semblaient les meilleures, mais d'essayer de découvrir si l'histoire providentielle, morale ou patriotique est légitime en soi. Pour délimiter la question, disons que nous cherchons à connaître, dans cet exposé, si les points de vue providentiel, moral ou patriotique, pour expliquer certains faits historiques, sont conformes aux lois de l'esprit - donc légitimes, - au même titre que les explications économique, politique, institutionnelle, etc., et, conséquemment, si l'on peut écrire ou enseigner de l'histoire providentielle, morale ou patriotique.

Les notions auxquelles nous ferons appel se retrouvent dans le livre du père Bernard Lonergan, s.j. Son livre est intitulé : Insight. A Study of Human Understanding. Il nous sera impossible d'utiliser ici toute la richesse de pensée que renferme ce volume. Nous nous limiterons à quelques notions de base, susceptibles d'apporter quelque lumière au sujet qui nous intéresse.

Pour comprendre le cheminement des idées qui vont suivre, jetons quelques jalons de base.

Dans la connaissance, il y a trois niveaux, à savoir : l'expérienciation, l'intellection et la réflexion. A ces trois niveaux, il faut en ajouter un quatrième, qui est la décision. Les trois premiers se rapportent à la connaissance; le quatrième, à l'action. Tout comportement raisonnable, donc humain, suit cette progression. Exemple : supposons le bombardement d'une ville. Vous êtes chez vous. Soudain, vous entendez un bruit grandissant, c'est l'expérienciation; l'idée vous vient aussitôt que c'est un avion, c'est le deuxième niveau : l'intellection; vous écoutez de nouveau et courez à la fenêtre pour être sûr, c'est la réflexion, faite, ici, de vérification, donc troisième niveau; vous laissez tout pour courir à l'abri le plus près, c'est la décision qui suit la connaissance, donc quatrième niveau.

Dans la connaissance historique, ce même schéma fondamental est suivi par tout historien. A l'expérienciation, correspondent les documents matériels ou écrits qui offrent des données historiques; à l'intellection, correspondent les idées et les hypothèses qui permettent d'établir les faits ou de les expliquer; à la réflexion, correspondent la discussion critique des hypothèses et leur vérification par des retours aux données. 
Exemple : l'exploit du Long-Sault. A l'expérienciation, correspond le contact avec les différentes sources qui traitent de cet exploit : la main rassemble la documentation, les yeux la lisent, l'esprit apprend ce qu'elle contient. A l'intellection, correspond la reconstitution de cet exploit à partir des différentes sources. Enfin la réflexion, c'est la vérification du reconstitué qui, par la preuve, devient affirmé : la réflexion, ici, s'exprime par l'appareil critique, par les références et, s'il y a lieu, par la discussion des points obscurs.

Ainsı, en historre comme en toute autre science, sont reconstitués les faits. Il y a d'abord les données, ensuite les hypothèses, puis leur vérification, après quoi les faits sont établis, pas avant. Ces faits établis par l'esprit sont reliés entre eux par la narration. Mais la narration historique n'est que de la description; elle se borne à faire voir comment les choses se sont passées. Elle est dite de sens commun, si l'auteur raconte les événements tels qu'il les voit, tels qu'il les a entendus, en termes usuels : c'est le cas des différentes sources, des témoins oculaires; elle est scientifique, si tout est vérifié et si les faits sont dégagés des différentes sources pour être reliés entre eux : c'est le cas des ouvrages savants.

Toute description est orientée vers l'explication. Une fois les faits établis et décrits, l'histoire doit, comme toute autre science, s'élever au second palier, c'est-à-dire à l'explication des faits. C'est ici que se place la multiplicité des points de vue supérieurs qui peuvent être dégagés des faits. Ces points de vue supérieurs seront, disons : l'économique, le politique, le militaire, le religieux, etc. Pour saisir ce point, reprenons notre exemple. Dans l'exploit du Long-Sault, ce qui a trait aux fourrures s'expliquera par le point de vue économique; ce qui a trait à l'arrêt des Iroquois, par le politique; ce qui a trait à la bataille par le militaire; ce qui a trait à la confession et à la communion des Français, par le religieux. Pour être valables, ces points de vue supérieurs doivent sortir des faits et être vérifiés par les faits. La démarche de l'esprit est la suivante : l'esprit abstrait des faits un point de vue, disons : le politique, ici, l'arrêt des Iroquois, c'est l'intellection; puis il passe à la vérification, en discutant et en prouvant que l'exploit de Dollard a bel et bien arrêté les Iroquois dans leur dessein de surprendre Ville-Marie, c'est la réflexion critique. Si l'on arrive à l'inconditionné, c'est-à-dire si toutes les conditions qui exigent l'arrêt des Iroquois sont remplies, ce point de vue supérieur est rationnel et, par là, valable, car il est conforme à la démarche de l'esprit dans sa connaissance des faits. L'esprit, par la preuve, a dégagé le conditionné de ses conditions, après quoi il l'a affirmé comme inconditionné. Il est scientifique parce que toutes les étapes qui mènent à l'inconditionné sont contrôlées par la réflexion critique.

Qu'il y ait hiérarchie des points de vue, que l'un domine l'autre, qu'il y ait finalité, c'est une autre question qui pénètre plus avant dans le problème. Nous ne l'aborderons pas ici. 
Les points de vue supérieurs entraînent la conséquence suivante. Le langage dans lequel s'exprimera l'explication ne sera plus le langage concret de la description des faits, mais celui de l'abstraction. Comme toute science a son langage abstrait, l'histoire a aussi le sien. Exprimons cette affirmation en d'autres termes; ce que l'algèbre et les différents calculs sont par rapport à l'arithmétique, les abstractions historiques le sont par rapport aux faits historiques, c'est-à-dire que les abstractions historiques permettent à l'esprit de raisonner rapidement avec des groupes de faits, comme l'algèbre permet de raisonner, par ses symboles, avec des groupes d'opérations arithmétiques. Les livres de synthèse historique sont de cette nature. Les discussions sur le paternalisme de l'Etat français au XVIII' $^{\circ}$ siècle, sur la bourgeoisie canadienne de 1760 , sur l'évolution de la noblesse au Moyen Age, et bien d'autres, sont également de même nature. En d'autres mots, les termes paternalisme, bourgeoisie, noblesse, représentent des groupes de faits concrets. Ce sont des séries de corrélations de corrélations de corrélations, qui ne peuvent être exprimées que par des abstractions historiques. Pour être valables, il faut que l'esprit les abstraie sans faille, à partir des faits ou des groupes de faits, et qu'il puisse, pour les vérifier, redescendre sans faille jusqu'aux faits, comme il le fait pour résoudre une équation algébrique.

Les langages concret et abstrait, descriptif et explicatif, soulèvent le problème des genres historiques. C'est une autre question, que nous n'aborderons pas ici.

Lorsque l'esprit abstrait des faits un point de vue supérieur, il laisse de côté les faits qui ne l'intéressent pas, ceux qui ne sont pas nécessaires. Exemple : l'économique tirera des faits historiques ceux qui peuvent être groupés et reliés entre eux sous ce point de vue. Ainsi en est-il du militaire, du religieux et de tout autre. Appelons ce qui est ainsi rejeté : le résidu historique. Dans tous les domaines scientifiques : physique, chimique, biologique, etc., l'esprit procède de cette façon : il y a toujours un résidu que l'on nomme le résidu empirique. Or, ce résidu est très important, car c'est vers lui que l'esprit se tourne pour abstraire de nouveaux points de vue supérieurs. La physique n'explique pas tout, il faut se tourner vers la chimie; celle-ci n'explique pas tout, il faut se tourner vers la biologie; celle-ci n'explique pas tout, il faut aller à la psychologie et ainsi de suite. Le résidu historique suit la même voie : le politique n'explique pas tout, il faut aller à l'économique, puis au militaire, aux institutions, à la psychanalyse, à la sociologie, etc.

Nous arrivons avec ces notions de base au cœur de notre question du début: l'histoire providentielle, morale ou patriotique est-elle légitime? Cette histoire est légitime, si elle abstrait du résidu historique des points de vue qui expliquent des faits inexpliqués par l'économique, le politique, le militaire, etc., suivant les mêmes démarches de l'esprit qui légitiment les points de vue économique, politique, militaire, etc.

Or, les documents écrits rapportent plusieurs faits où Dieu apparaît comme intervenant de façon spéciale dans le cours des événements et 
révélant des choses qui dépassent la capacité de l'intellection humaine. C'est le cas d'Abraham, des prophètes, de Jésus-Christ, des voyants et voyantes de l'Ancien et du Nouveau Testament. Après ces interventions, le cours des événements a été changé. Beaucoup d'hommes ont agi suivant les révélations faites par Dieu. Les institutions humaines ont été modifiées, des civilisations entières s'en sont imprégnées, les faits en portent la marque. Comment relier entre elles toutes ces choses, comment expliquer tous les changements encourus par ces interventions de Dieu, si l'on ne fait pas intervenir le point de vue d'une providence surnaturelle? Tous ces faits sont laissés dans le résidu historique par les autres points de vue : économique, politique, diplomatique, etc. L'esprit peut donc, tout aussi bien que pour ces autres points de vue, relier entre eux les faits qui dépendent d'âge en âge de ces interventions providentielles, comme des cascades de causes et d'effets.

Il en est de même du point de vue moral. Il y a quantité de documents dans lesquels se reflètent les décisions des hommes. Ceux-ci ont agi rationnellement ou irrationnellement, et le cours des événements en a porté la marque : régimes viciés, civilisations décadentes, révoltes, assassinats, vols, etc. La matière en ce domaine est abondante dans les documents. Au fond de l'histoire, il y a l'homme avec son mélange de bien et de mal, l'homme qui charpente ses systèmes et sa cupidité qui les vicie. Comment expliquer ces faits rationnels et irrationnels, si on ne les relie pas à la moralité de leurs auteurs?

Ainsi en est-il du point de vue patriotique qui n'est, au fond, qu'une subdivision du point de vue moral. Des hommes ont agi par amour pour leur patrie, par fidélité à leur culture, par esprit de dévouement envers le groupe humain auquel ils appartenaient. Les gestes et les faits qui en ont découlé ne peuvent s'expliquer que par le point de vue patriotique et, par lui seul, être reliés à leurs auteurs.

Dans ces trois points de vue : providentiel, moral et patriotique, l'esprit suit les mêmes voies que dans les autres points de vue : économique, politique, militaire, etc. Il abstrait son point de vue du résidu historique, c'est l'expérienciation; il relie entre elles les choses qui s'y rapportent, c'est l'intellection; il les vérifie dans les faits et tend à l'inconditionné, c'est la réflexion critique. Donc, ces points de vue providentiel, moral et patriotique sont légitimes au même titre que les points de vue économique, politique, militaire, etc.

Que ces points de vue soient parfois difficiles à dégager, qu'ils donnent plus de prise à des erreurs d'interprétation, cela n'implique pas qu'il soit illégitime de les considérer. Il y a eu des erreurs dans l'élaboration de toutes les sciences; c'est par la correction de ces erreurs que les sciences ont pu arriver à se systématiser. Autrefois les rêves étaient inexplicables. Depuis Freud, la psychanalyse a fini par trouver le moyen de les expliquer. L'histoire, il n'y a pas si longtemps, ne s'occupait que du politique, du militaire, du religieux. Aujourd'hui, le champ s'est élargi; on fait l'histoire des institutions, des sociétés, des sciences; on peut faire l'histoire de la maison canadienne, des costumes, 
des professions libérales, etc., points de vue qui, autrefois, étaient laissés dans le résidu historique. Pour pénétrer plus avant, on utilise la sociologie, la caractériologie, la psychanalyse, comme autant de cadres référentiels pour éclairer notre connaissance des sociétés passées et des individus. Au même titre, on peut également se servir de la théologie providentielle, de la théologie morale ou simplement de la morale naturelle, des intérêts ou des formes de culture de son pays, pour éclairer l'histoire providentielle, morale ou patriotique.

Parfois, des historiens expliquent les faits en se servant, ensemble ou tour à tour, des différents points de vue supérieurs, comme le musicien qui joue sur différentes gammes à la fois. Cette façon de faire n'invalide en rien ces points de vue supérieurs, si leurs corrélations sortent des faits et si elles se vérifient en eux. Elle relève des genres historiques, de la façon de communiquer à d'autres les propres intellections de l'auteur. Par contre, beaucoup d'historiens, formés aux rigueurs scientifiques, préfèrent livrer leurs intellections dans la logique exclusive d'un point de vue à la fois. Les grands avantages qu'il y a à le faire sont universellement reconnus.

Ajoutons un corrollaire à ce qui précède, en posant la question suivante : peut-on tirer de l'histoire des leçons providentielles, morales et patriotiques?

Pour répondre à cette question, distinguons deux choses : la science pure et la science appliquée. La science pure découle du pur désir de connaître et son objectif est l'inconditionné; la science appliquée part des trouvailles de la science pure et elle les fait servir à des fins pratiques. La science pure se situe aux trois premiers niveaux de la connaissance : l'expérienciation, l'intellection et la réflexion critique; la science appliquée se situe au quatrième niveau : la décision. Pour agir rationnellement, c'est-à-dire en toute connaissance de cause, l'homme doit d'abord connaître la vérité dans toute sa rigueur, et c'est en passant par les trois premiers niveaux que ce but est atteint. Par eux, l'homme arrive à la preuve, à l'inconditionné, il atteint le réel, la vérité, le vérifié. Donnons un exemple tiré de la physique : l'hydrostatique. Le physicien, en science pure, déterminera les lois de la portabilité de l'eau, de la densité des corps; l'ingénieur, en science appliquée, utilisera ces lois dans la construction navale et fera en sorte qu'un navire, pour porter sur l'eau, se conforme à ces mêmes lois.

Cette distinction n'est pas toujours aussi claire pour toutes les sciences qu'elle l'est pour la physique, la chimie, la médecine. Certaines sciences ont beaucoup tardé à développer les techniques de leurs applications pratiques, par exemple : l'astronomie. Cette science, très ancienne dans ses origines, n'a vu que de nos jours se développer sa branche pratique qui est l'astronautique, avec ses projets de vols interplanétaires. Cependant le principe de cette distinction est toujours sous-jacent à toute science, c'est-à-dire que l'homme agit après avoir expériencé, intelligé et réfléchi. 
Il ne saurait en être autrement pour l'histoire. Il y a la science historique pure; il y a la science historique appliquée. La science historique pure recherche la vérité des faits et des explications et son objectif est l'inconditionné, la vérité dans toute sa rigueur; tandis que la science historique appliquée utilise les trouvailles de la science historique pure et les applique à des fins pratiques. C'est ici que se placent les leçons tirées de l'histoire : l'histoire utilisée comme moyen d'éducation, comme moyen d'orienter la politique d'un pays, comme moyen d'orienter les institutions, comme moyen d'orienter la conduite personnelle des individus. Ce que les historiens de la science pure ont établi comme vrai sert aux hommes pour orienter leurs pensées et leurs actions, pour imiter les bons exemples, pour éviter les mauvais. En tant que science appliquée, l'histoire peut donc être mise au service de l'éducation, des institutions, des individus.

Qu'il y ait eu des erreurs, qu'il y ait eu de fausses applications de l'histoire, des sermonnages inopportuns et mal conçus, des envolées patriotardes plutôt que patriotiques, nul ne le contestera. Toutes les sciences appliquées ont connu leurs erreurs : on a vu des bateaux mal équilibrés qui prenaient l'eau, des avions qui ne volaient pas, des fusées qui éclataient au lieu de prendre leur essor, comme on a vu des applications historiques absurdes à l'instar du surhomme hitlérien. Ces erreurs n'infirment en rien la distinction entre science pure et science appliquée.

Cependant deux grandes difficultés, entre autres, se présentent aux historiens de ces deux sciences. D'abord, tandis qu'en physique et en chimie les faits sont toujours présents et renouvelables à volonté, les faits humains, eux, au moins les faits individuels, ont été posés une fois pour toutes et ils s'enfuient sans cesse vers un passé de plus en plus lointain : pour les reconstituer, l'historien doit remonter dans le temps et travailler sur des matériaux souvent incomplets. Deuxièmement, les méthodes des applications de l'histoire n'ont pas encore été suffisamment étudiées et systématisées, alors que dans d'autres domaines, on s'y applique déjà depuis des siècles. Les individus sont laissés à eux-mêmes dans ces applications pratiques, comme ce fut le cas au début de la physique et de la chimie.

\section{CONCLUSION}

L'histoire providentielle, morale ou patriotique est-elle légitime ? Nous ne pensons pas que le problème qui se pose à nous aujourd'hui ait sa solution dans le rejet ou dans la négation de la légitimité des différents points de vue et dans la négation des applications pratiques. Il s'agit plutôt d'un problème de réadaptation constante, sous l'effet de l'accumulation du savoir et de la multiplication des points de vue. Ce qui paraissait, hier, acquis de façon sûre est, aujourd'hui, désuet. Les trouvailles historiques qui servaient aux applications pratiques doivent être modifiées par suite d'une meilleure connaissance du passé. Le même phénomène se rencontre d'ailleurs dans les autres domaines scien- 
tifiques. Il n'y a plus d'équilibre stable, mais un équilibre dynamique, qui résulte d'une marche sans cesse plus rigoureuse vers la vérité, d'un savoir qui élargit sans cesse ses horizons. Comme en cybernétique qui est bien une science de notre temps où, dans tous les domaines, l'équilibre est de plus en plus instable, - l'historien doit accepter de faire sans cesse le point et de réajuster ses positions.

Pour résumer : nous ne pensons pas qu'il faille nier ou rejeter les points de vue providentiel, moral ou patriotique qui ont eu cours depuis des siècles, mais nous croyons qu'il faut, en science historique pure, travailler à découvrir de nouvelles méthodes pour mieux les traiter et s'efforcer, en science historique appliquée, d'élaborer de meilleurs modes d'application pratique.

Jean LECLERC, s.j.

Résidence des Jésuites, Québec, P.Q. 